\title{
Interactive Learning: Developing an eSimulation Portal Framework
}

Research Article

Ayman Tobail, John Crowe* and Amr Arisha

3S Group, College of Business, Dublin Institute of Technology

Aungier Street, Dublin 2, Ireland

\begin{abstract}
The emerging eLearning paradigm is becoming increasingly used in many academic disciplines. The concept acknowledges learning processes that no longer deploy only traditional teaching methods. It can be argued that today's third level education students are part of a new virtual era where the blackboard has been replaced by the interactive whiteboard, so more interactive and virtually orientated teaching aids are required to assist in the transition from traditional learning to eLearning. The key objective of this paper is to propose a simulation-based learning framework that integrates web-based simulation and a web content management hierarchy model. Using the highly complex subject of supply chain management as a field of study, the newly developed eSimulation tool allows users to examine various management strategies in real-life scenarios, encourages group work and offers remote access capabilities for distance learning. Interactive learning is facilitated using a web-based portal, enabling instructors to demonstrate the complexity of decisions in multiple criteria environments and also demonstrate the impact of various strategies on performance to the users. eSimulation creates an animated experience and better understanding of the system dynamics of decision-making processes. The portal interface is friendly and, hence, has the potential to be applied in other subject areas.
\end{abstract}

Keywords: eLearning • simulation • supply chain management education

(C) De Gruyter Open Sp. z 0.0.

\section{Introduction}

The ability to learn, which can be defined as the acquisition of knowledge through cognitive processes that translate into new understandings, behaviours and skills (Moore et al., 2009), has always been the foundation of any successful society. In today's knowledge-driven society, such valuable understandings, which can be gained through education, are very important resources (Schleicher, 2003). The advances made in computer technology, coupled with the education sector's drive to take advantage of such advances, have given rise to the emerging eLearning paradigm, which is becoming increasingly evident across many academic disciplines, providing further support for the notion that learning processes are no longer only supported by traditional teaching methods. It can be argued that today's third level education (TLE) students are part of a new virtual generation, where blackboards and refill pads have been replaced by interactive white boards and laptops, as more interactive, animated and virtually orientated teaching aids assist the transition from traditional learning to eLearning.

The main contribution of this paper is to create a framework to enable the examination of different innovative teaching technologies such as simulation through a web-based portal accessible from anywhere. First, we give an overview of TLE and its challenges from an Irish perspective. Second, we discuss innovations in teaching/ learning processes, with particular emphasis on simulation-based applications, using the complex field of supply chain management as our study field of knowledge. The advantages of utilising web-based technologies are then reviewed before the methodology of our portal framework is introduced and discussed in detail. The paper concludes with a conceptual overview of the portal framework, and outlines its potential future development into to a serious working TLE game. 


\section{Research background}

\section{Third level education}

It is widely recognised that the future economic success and social well-being of countries is closely linked to the level of their populations' knowledge and skills (Breena et al., 2009). The effectiveness of TLE is extremely important to allow Ireland to gain the levels of knowledge and skills required to build its knowledge economy, so Irish governments have put increased emphasis on higher education over the past few decades, which resulted in a major increase in the number of Irish TLE students (Figure 1) (Skills, 2012). In particular, government incentives such as the abolition of college fees in 1996 (Clancy and Kehoe, 1999), and the 1995 'Charting Our Education Future' white paper (Education, 1995) laid the foundations for education for the Irish knowledge economy. However, given such a high level of investment in TLE, it is critical that the challenges involved in the teaching/learning relationship are understood and addressed to optimise college graduates' successful transitions into the workplace (Tobail et al., 2010).

Figure 1. Enrolments of full-time students in Institutions aided by Department of Education, Ireland (Source: Irish Department of Education, 2012)

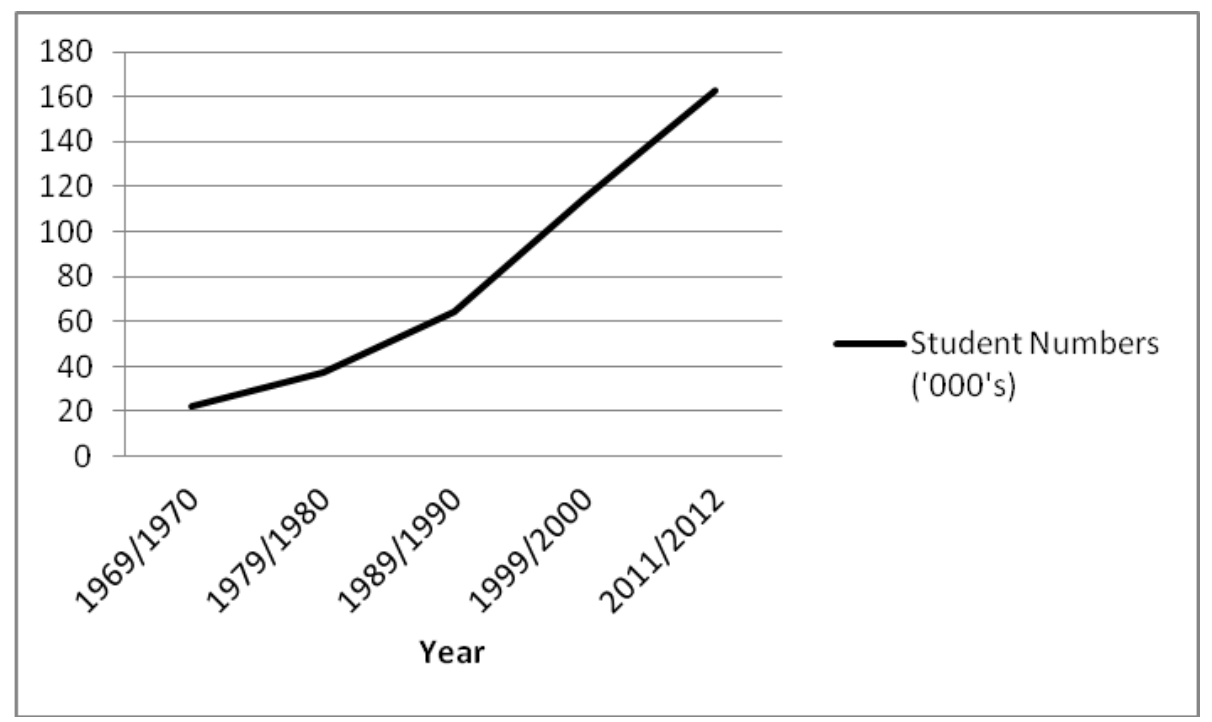

\section{Challenges to the teaching learning process}

The relationship between TLE teaching and learning hangs on a delicate balance between the student's willingness and ability to learn and the lecturer's willingness to create an effective learning environment: maintaining a successful balance can make all the difference to students' TLE learning experiences (Palmer, 2010). Doing this effectively has required a move away from more traditional TLE teaching methodologies. In traditional teaching, the success of college graduates was often predicted by the amount of knowledge they had accumulated during their degree courses (Knight and Wood, 2005). This suggests that a lecturer in the traditional setting informed students rather than transformed them (O'Neill et al., 2005), and that traditional university courses were characterised by a lack of flexibility in terms of their teaching content (Rainer and Guyton, 1994). Traditionally, teaching was fundamentally conceived of as information transfer between the teacher and the student only. Academics in the past typically believed this learning process consisted of knowledgeable educators, who were experts on particular topics, constructing and communicating knowledge on those topics to learners, using the common instructional technologies of the day - books, articles and classroom lectures (Ruben, 1999). As early as the 1950s, Skinner's studies suggested such 'rote learning' was outdated (Skinner, 1954). In fact, it was argued back in the late 1930s that effective education is based on the interaction of an individual's external and internal environments, also known as constructive learning (Dewey, 1938). Constructivist learning activities are characterised by active engagement in classroom processes, collaboration with others, inquiry, reflective thinking and problem solving (Von Glasersfeld, 1989; Savery and Duffy, 1996). 
During the past three decades, TLE has experienced a revolution, and the objectives of third level institutes have changed. Memorising facts and figures is now recognised as less important than developing knowledge-based skills for problem-solving, interactive team work and life-long learning (Knight and Wood, 2005). The introduction of the learning pyramid has instilled a new focus on how teachers interact with students in terms of how well they retain what they are taught (DeKanter, 2004). In brief, the pyramid suggests that over $90 \%$ of all the learning students retain is what they use immediately in practical terms, and then teach back to each other in group work sessions and presentations (O'Neill et al., 2005), but that only $5 \%$ of what they retain is taught by traditional 'rote learning' class lecture processes. The foundations of the framework developed in this paper are built on the concept of this pyramid.

\section{Innovations in the teaching learning process}

Learning can be seen as an iterative process loop, the ongoing refinement of a conceptualise-construct-identify pattern, with dialogue playing a central role in each stage (Fowler and Mayes, 2000). Laurillard (1998) articulated the ideal teaching and learning process as an iterative four-stage sequence model, as shown in Table 1. An important emphasis is placed on the key elements of understanding the practical aspects of what is learned in theory - that is, discussing what is learned, interaction within the class about what that learning means, adapting that knowledge for greater understanding and reflecting on the learning outcome achieved to further improve the learning process. Active learning, as discussed by Prince (2004), echoes the same theme of an interactive, collaborative and cooperative approach to learning. Problem-based learning (PBL) is another technique that also allows students to interact with theoretical problems in more practical, real life, ways, using the ideology of active learning, and has been especially employed in medical school settings (Prince, 2004; Chan, 2009). Stefanou et al. (2013) noted that active learning is the basic assumption behind self-regulated learning (SRL), in which students actively participate and construct meaning from available information.

Table 1. Characteristics of the ideal teaching/learning process (Laurillard, 1998)

\begin{tabular}{ll}
\hline Process Stage & Characteristic \\
\hline \hline Discussion & Between teacher and learner at the level of descriptions \\
Interaction & Between the learner and some aspect of the world defined by the teacher \\
Adaptation & Of the world by the teacher and action by the learner \\
Reflection & On the learner's performance by both teacher and learner \\
\hline
\end{tabular}

Overhead projectors and PowerPoint slide presentations are the most commonly used technologies in teaching. But there are also more complex methods such as distance learning, online and web-based learning and a combination of information technology with traditional teaching called 'blended learning', which, at its basic level, integrates face-to-face classroom learning with online learning (Garrison and Kanuka, 2004). Wireless keypads (Burnstein and Lederman, 2001) and audience response systems (ARS) or clickers (Caldwell, 2007) have also aided in the transformation of the third level lecture, and the use of simulation technologies is becoming an accepted method of teaching theory with a practical edge. Medical, nursing and engineering schools have been at the forefront in advancing this learning process (Ferdig et al., 2007; van der Zee and Slomp, 2009), but simulation also shows encouraging potential for use in business education, especially in areas such as supply chain management.

\section{Simulation as an educational technique}

Although using simulation as a teaching method is not a new concept, particularly in medical, military and aviation settings (Murphy et al., 2011), its use is growing rapidly in many other academic disciplines because of its potential to create experiences that closely mimic real-life scenarios (Zhang et al., 2010). Whether simulating medical procedures without risking doing harm to patients, or simulating a supply chain management concept without the costly change in business strategy required to be experimented in real-life settings, simulation can be a powerful learning aid. Another important driver in the growth of the use of simulation technologies in TLE is the fact that today's TLE students are part of the digital generation. They already live in a virtual age, where online multi-player games, virtual reality and simulations (such as Warcraft and Second Life) are a part of everyday life, making gaming and simulation very important catalysts in the TLE learning process (Ferdig et al., 2007; Proserpio and Gioia, 2007). 
As noted, textbooks and case study literature are limited as teaching aids, and are insufficient to transport students into the positions of managers who make complex decisions every day (Katsaliaki et al., 2014). Supply chain management (SCM) can be a very effective setting in which to demonstrate the advantages of simulation as an education technology.

\section{Supply chain management}

Although the concept of SCM was first introduced in early 1980s by Oliver and Webber (cited in Jüttner et al., 2007), it did not come to prominence until the mid-1990s. As a management philosophy, it is a vast concept, with many interpretations and definitions. SCM can be defined as the efficient and effective management of a firm's upstream and downstream relationships (i.e. with its suppliers and customers) in order to create enhanced value in the final marketplace at less cost to the firm and to its supply chain as a whole (Christopher, 2010). Figure 2 illustrates the basic supply chain relationships between upstream and downstream partners; information (the order cycle) flows in both directions, materials flow downstream towards the end user, whereas capital flows upstream to finance the chain's operations at various points. A series of strategic collaborations between business partners work via close supply chain relationships to bring greater value to the customer (and thus to the end consumer) for the least possible supply cost (Hung et al., 2004).

Figure 2. Simple Supply Chain Model

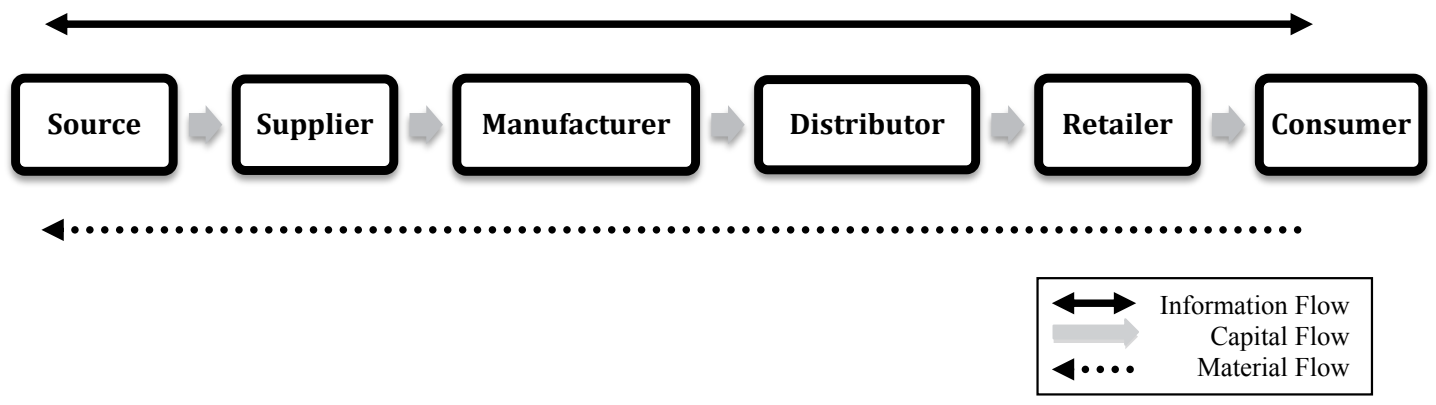

\section{Supply chain challenges}

At its basic level, a supply chain is made up of multiple actors and multiple flows of items, information and finances and is sometimes described as looking like an uprooted tree (Lambert and Pohlen, 2001). Each network node has its own customers' and suppliers' management strategies, partnerships, demand arrival process and forecast methods, inventory control policies and item mixtures (Longo and Mirabelli, 2008). There are many challenges to be overcome at all strategic SCM levels, including complexity, uncertainty, risk, resilience, visibility and cost, to name a few.

This supply chain complexity is the key managerial issue that SCM needs to address, especially in terms of operational processes and manufacturing strategies (Caridi et al., 2010), and can be very hard for TLE students (as potential future SC decision makers) to visualise. The complexity of most supply chains makes it difficult to understand how the actions and interactions of multi-tier supply chain partners influence each other (Lambert and Pohlen, 2001).

\section{Supply chain uncertainty}

Supply chain uncertainty, most notably the uncertainty between supply and demand, is a key issue known to impact supply chain effectiveness (Davis, 1993). Examples of uncertainty in the supply chain process include demand quantities, sales orders, delivery/arrival times, suppliers' lead times and defective rates of received products (Crowe et al., 2010). Andrew Grove, a former president of Intel, suggested that research into supply and demand at the company found that they were in equilibrium for just 35 minutes in 10 years (Towill, 1991; Wilding, 1998; Huin et al., 2002). The complexity triangle developed by Wilding (1998) is a framework that can explain such uncertainty in the supply/demand relationship. The triangle explains that there are three interacting but independent effects that cause 
the dynamic uncertain nature of supply chains: deterministic chaos, parallel interactions and demand amplification. These effects resemble the uncertainty in decision making situations described by Van der Vorst and Beulens (2002), primarily, when decision makers cannot make definitive decisions because of a lack of information, process knowledge and behavioural impacts and controls.

\section{Supply chain visibility}

Information and material flows (order cycles) play very important roles in the effectiveness of SCM, both upstream and downstream along the supply chain. This order cycle is often referred to as pipeline time, and confidence in the supply chain is weakened if the pipeline time is too long (Christopher and Lee, 2004). Visibility of material and information flows are associated with the length of the pipeline time, and the key to improved supply chain visibility is better sharing of information along the supply chain (Christopher and Lee, 2004). Collaboration through the entire supply chain is needed to create a transparent, visible demand pattern that paces the entire supply chain (Holweg et al., 2005).

\section{Supply chain collaboration}

Collaborative links allow stronger relationships to form along the supply chain which, in turn, will drive competitive advantage for its partners (Spekman et al., 1998). The days of poor co-operation where suppliers are kept at arm's length, as in the traditional open-market style relationships outlined by Cousins (2002) are gone, and a new wave of collaboration among firms is being developed based on high levels of trust, commitment and information sharing (Spekman et al., 1998). Figure 3 illustrates how this collaboration moves through the supply chain relationships

Figure 3. Transition from Open Market Negotiations to Collaborative Partnerships

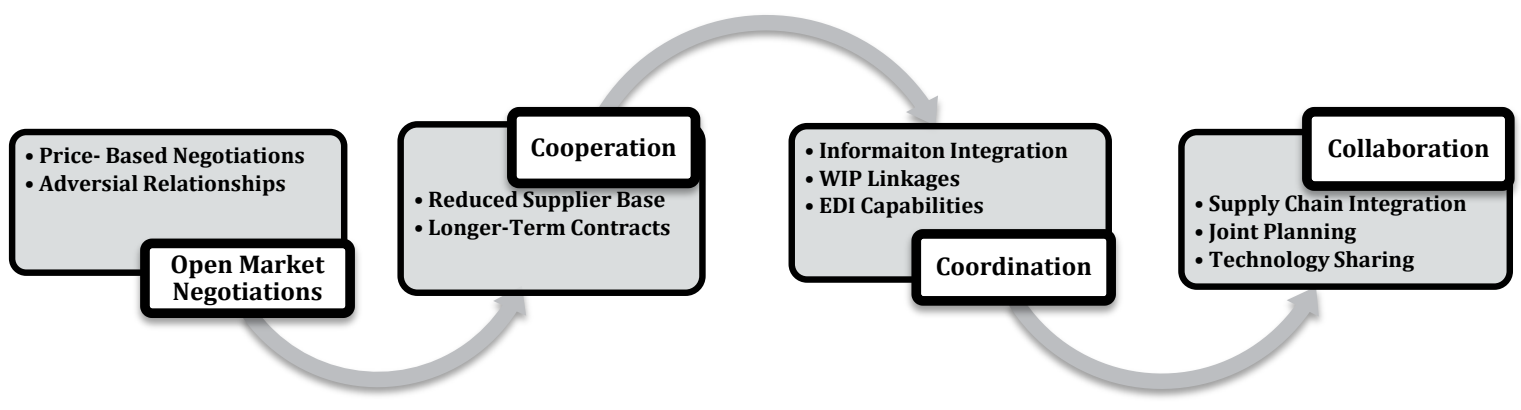

SCM is about the management of relationships across complex networks. Successful supply chains will be those that are governed by a constant search for win-win solutions based on collaboration, mutuality and trust (Christopher, 2005). Cousins (2002) outlined four distinct relationship types as being most effective for win-win relationships, depending on the level of strategic collaboration needed: traditional, opportunistic behaviour, strategic collaboration and tactical collaboration.

\section{Supply chain simulation}

Simulation software is a very powerful tool in highlighting the importance of collaboration to SCM, while also educating students on the high levels of risk involved due to supply chain complexity. A supply chain is stochastic and dynamic in nature, resulting in high levels of variability and uncertainty. Simulation software has high capabilities to replicate uncertainty, mainly through discrete event simulation, which is capable of manipulating a system's variability and uncertainty (Mahfouz et al., 2010). However, there have been very few examples of SCM simulation theory being used in TLE, the most popular being the 'beer game' introduced by MIT in 1960 as an exercise in industrial dynamics (lyer et al., 2009).

Some academics say that gaming and simulation have been under-utilised by educators, with institutes focusing on their negative social associations, but ignoring their important potential as teaching aids (Squire, 2003). Little has changed since the beer game's introduction: there have been some advances in simulation education such as van der Zee and Slomp's (2009) assembly line simulation game and the activity-based-costing (ABC) flash simulator 
game developed by McKee and Lantz (2009). Although very effective in visualising and simulating the fields of production processes and costing, these examples lack the scope to incorporate all supply chain areas. Simulation has huge potential to be a very effective tool in teaching practical SCM operations: Figure 4, adapted from Bond (2002), illustrates how it can be used as a link between the active learning of constructivism and the hands on experience of real-life practice.

Figure 4. Simulations Link between Theory and Real-Life Practice

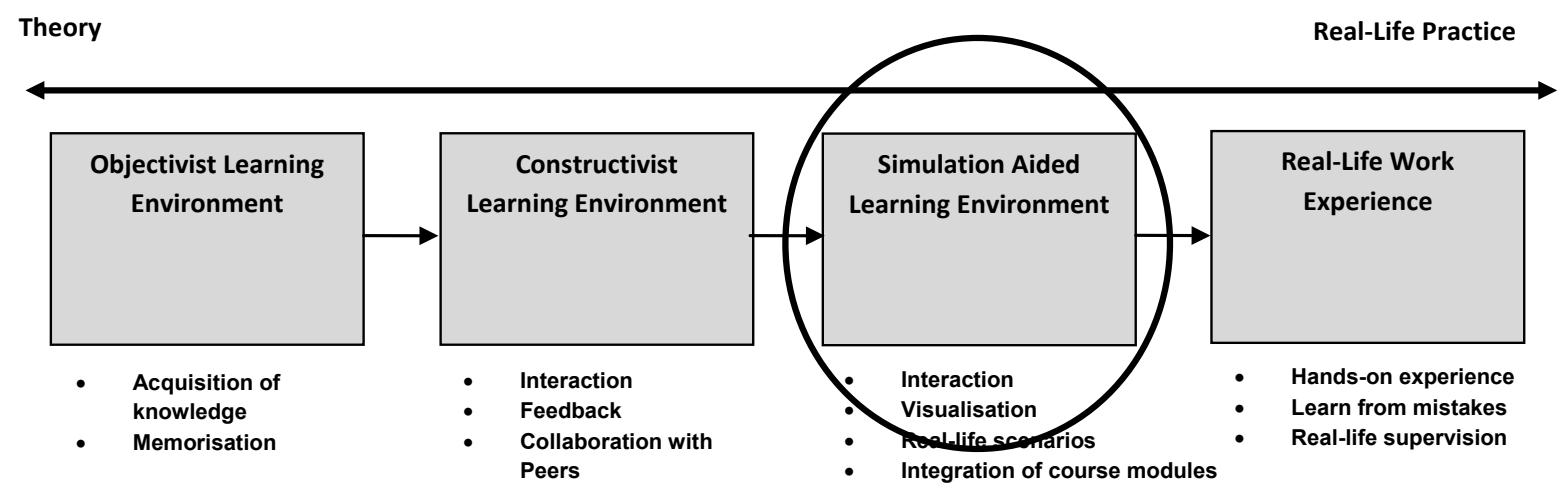

\section{Teaching supply chain management using simulation technology}

The central aim of this paper is to investigate the potential of using interactive simulation technologies to facilitate the learning of SCM concepts. Simulation educational innovations such as the beer game and the $A B C$ flash simulator have been found to be very effective in aiding teaching about of certain tiers of supply chain networks, such as distribution, material movement and costing. However, they lack the fundamental ability to visualise and demonstrate the operations of the whole supply chain effectively, starting from the source of raw material to the final delivery to the end consumer. As noted, the importance of collaboration and visibility along the supply chain is self-evident, and these simulations do not incorporate this factor, although some simulated supply chain models have been developed that do incorporate a broader scope, such as (Longo and Mirabelli, 2008) SCM decision support tool and (Rossetti et al., 2008) object-orientated framework for simulating supply systems.

But it is important to note that these models were developed as analytical decision-making tools for supply chain managers, and do not have the interaction, animation or academic attributes required to stimulate the minds of TLE students. Web-enabling technologies can also be powerful educational tools to encourage collaborative learning about SCM. The recent emergence of online technologies such as social networking reflects the potential improvement in interaction between TLE students that high-quality virtual environments can offer (Davis et al., 2007). It has been argued that utilising web technology is an obvious choice when implementing new study materials to liven up lectures (Syrjakow et al., 2000), which is an obvious advantage in a sometimes very analytical subject such as SCM. Web-based education games also have the advantage of being much cheaper than expensive simulation software, and can also give students online access outside the classroom (Tobail et al., 2010).

\section{Web-based learning}

The increasing demand for education (Figure 1), coupled with the growing amount of information available, are the main reasons for the integration of the use of computers, primarily via the internet (Bicen et al., 2010), into education. This novel approach, commonly known as web-based learning, is an eLearning technique that has made the learning process more accessible by stretching spatial and temporal barriers (Khalifa and Lam, 2002). Webbased learning is a step in the learning/teaching relationship, where students' communication and interaction with their lecturers and teachers takes place with the use of computer science and network technologies. 


\section{Web-based simulation}

A web-based learning field that has been growing steadily over the past number of years is that of web-based simulation (Yingping and Madey, 2005): although first introduced by Fishwick (1996), the concept is said to be as old as the web itself (Reichenthal, 2002). Fishwick's (1996) paper gave an introductory overview of web-based simulation, intended as a backdrop to a more formal discussion, with the objective of potentially forming a new simulation track that, in turn, gave rise to a new era in simulation study. Research into the field grew rapidly, but despite this promising start, the number of real applications in the field has remained relatively small (Wiedemann, 2001). According to Byrne et al.'s (2010) review, web-based simulation can be separated into seven categories:

1. Local simulation and visualisation

2. Remote simulation and visualisation

3. Hybrid local/remote simulation and visualisation

4. Web-based simulation documentation

5. Web-based simulation model repository

6. Component-based simulation in relation to Web-based simulation

7. Distributed simulation in relation to Web-based simulation

Incorporating web content management, the portal developed in this paper lies in the final category of distributed simulation in relation to web-based simulation. In theory, all web-based simulation can, to some degree, be regarded as distributed simulation (Page et al., 1998). In such systems, the model designer should not need knowledge of the technical details used by the system creator to produce distributed simulations (Page and Opper, 1999). This is an important factor in the development of the distributed simulation portal reported in this paper, the goal of which is to develop a simulation portal that is widely accessible to TLE students, many of whom are not technically minded.

\section{Web content management}

Web content management (WCM) is defined as an organisational process (aided by computer software tools) for managing web content, which has a life cycle that runs from formation to destruction (Vidgen et al., 2001). In basic terms, WCM is an infrastructural support management system for websites that supports three main roles: those of the writer, the reader and the collection manager (Rein et al., 1997). To manage these roles in the simulation portal developed in this paper, we used McKeever's (2003) four-layer WCM hierarchy, which reflects the interacting WCM layers of content, activity, outlet and audience (Figure 5).

Figure 5. WCM Four-Layer Hierarchy

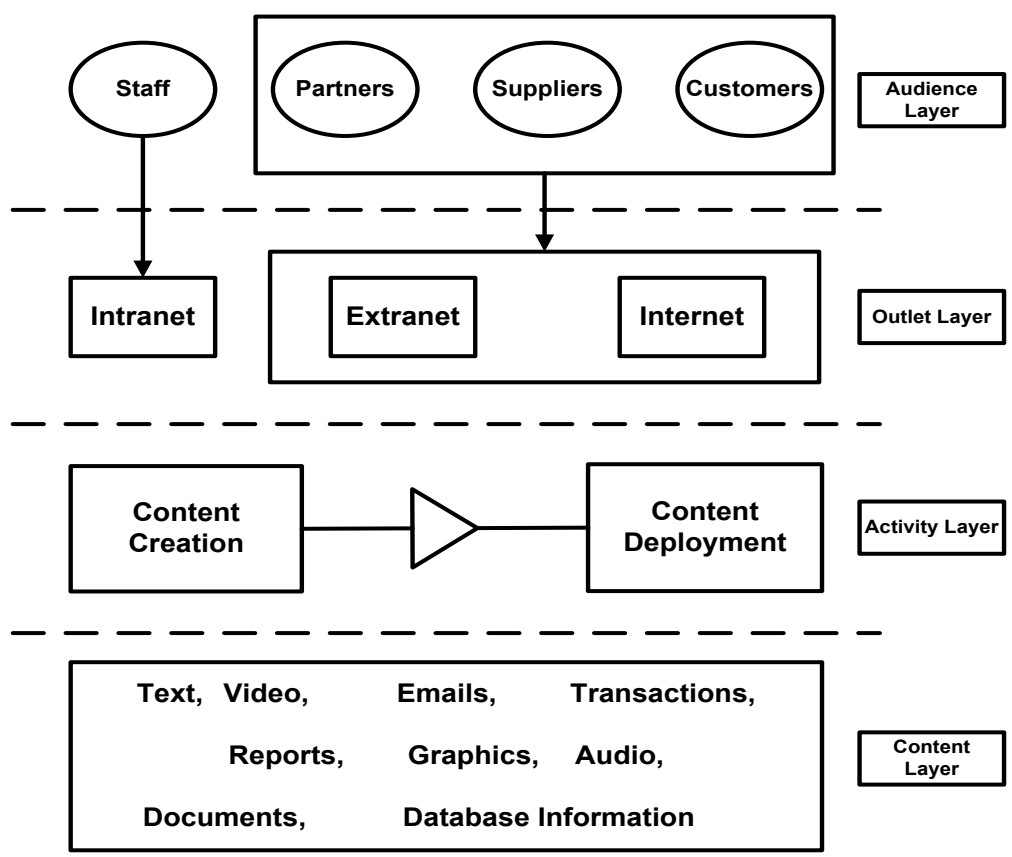




\section{Methodology}

Simulation is not a technology; it is a technique to replicate the real world in a completely interactive way (Gaba, 2007), but technologies such as computer software are often utilised to aid its effectiveness. This paper has discussed the benefits of using simulation models to aid TLE teaching, for which the powerful interactive benefits of web-based applications are also valuable. Figure 6 illustrates the web-based simulation or eSimulation framework developed in this paper.

Figure 6. eSimulation Portal Framework

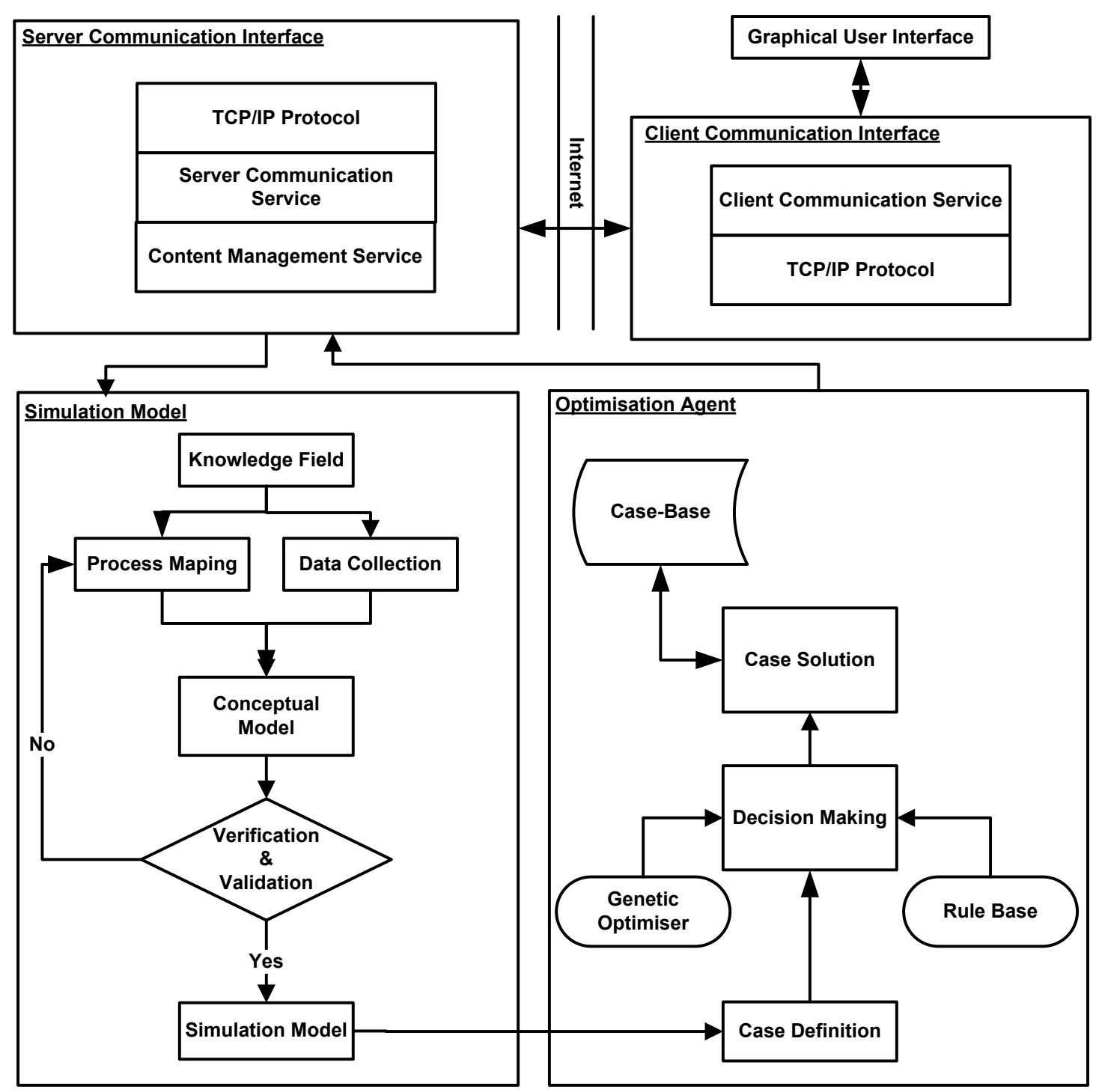

\section{eSimulation portal framework structure}

Using detailed conceptual models of a supply chain, the framework was developed to assist in creating a simulationbased teaching aid for TLE lecturers teaching in the SCM knowledge field. The whole framework depended on modelling the basic concepts and theories of SCM, and then integrated them into a powerful simulation tool. Designing and implementing this project's process involved using computer engineering and SCM experts to model the required aspects of the system accurately. Creating the framework can be divided into three stages. 
The first stage was the design and implementation of a SCM simulation model using a powerful simulation tool to output optimum potential solutions to help users in their decision making. The second stage was the creation of an optimisation agent that was integrated into the simulation tool to assist users in making optimal decisions. The third stage was developing web-enabling applications, using a communication protocol layer and interactive graphical user interface (GUI), to facilitate students using the simulation tool.

\section{Discussion}

\section{Simulation model}

The introduction to this paper highlighted the fact that education is a very important resource in today's knowledgedriven society: in the same way, knowledge is a very important resource in understanding and managing supply chains (Lambert et al., 1998). As noted, there are challenges to be overcome at all strategic SCM levels, including complexity, uncertainty, risk, resilience, visibility and cost and simulation software has high capabilities in replicating SCM uncertainty.

\section{Knowledge field: supply chain management case studies}

To illustrate how TLE students can learn about the complexity of SCM visually and interactively, two important network nodes - a distribution centre and a manufacturing plant - were chosen as the case settings to build the simulation portal. We studied these settings carefully, and used a powerful simulation tool to build two simulation models to be run on distributed server machines. The distribution centre's inputs, outputs and processes were linked to the corresponding parts of the manufacturing entity to form a supply chain relationship. Based on this framework, we designed and implemented a web-based portal to link the two simulation models and give users the facility to control, display and manage the relationships between them. A communication protocol was designed to allow the two models to be run as one supply chain (even if they run on distributed machines) via a single portal, so users could deal with them as if they run on their own machines. (This protocol could also enable the addition of further entities to the supply chain.)

\section{Distribution centre/manufacturer relationship}

Until the turn of the last century, relationships between supply chain partners tended to be adversarial in nature. In particular, supplier/manufacturer relationships were traditionally transactional, focusing solely on cost and delivery time (Goffin et al., 2006). To demonstrate the effectiveness of the simulation-based learning framework in teaching SCM complexity, two key supply chain entities - a first-tier supplier distribution centre and a manufacturer - were modelled conceptually, simulated and measured.

We studied the relationship between the two supply chain members using accurate input data, including forecast and actual sales figures, production process capacity, product specifications, lead-times and product costing. Equations, management strategies, statistical analysis and other management science techniques that SCM students will learn in their degree studies were integrated into the model results to illustrate the impacts of input and process decisions on the model outputs, which included warehouse capacity utilisation, cycle throughput time and queue lengths. The end objective was for the user to understand the complex relationship between the manufacturer and supplier in practical terms, and how the impact of their management choices and input decisions could affect the efficiency of the partnership.

\section{Data collection}

An important factor that affects the quality of a simulation model is the accuracy of input data (Ismail et al., 2010). System understanding and process analysis using qualitative and quantitative data collection methods were used in the analysis stage through the collection of primary data from both distribution centre and the manufacturer. We conducted several field visits, interviews, enterprise resource planning (ERP) software data collection and process analysis sessions to frame our understanding of the main parameters involved, and generate a list of the studied companies' SCM activities.

\section{Conceptual modelling}

Conceptual modelling is a presentation of the sequences of system processes, procedures and resources and shows the relationship between the system's objects such as customers and products and their status during the 
system processes (Mahfouz et al., 2010). Many modelling methods have been developed, studied and reviewed, from simple flowcharts to more complex object-orientated models (Aguilar-Savén, 2004; Shen et al., 2004). It was important to choose the best fit conceptual models for each process involved, and to capture the complex, dynamic nature of supply chain processes and decisions, so we used integrated definition modelling language for functional process (IDEF0) to develop the simulation-based framework.

IDEF0 is a structured graphical modelling form based on the structured analysis and design technique (SADT) (Ross, 1977; Ross and Schoman Jr, 1977), and is used to model decisions, actions and activities for manufacturing organisations and enterprises, so as to help the modeller identify and analyse the functions being performed and its requirements to perform. Colquhoun et al. (1993) have discussed various applications of IDEF0, and Busby and Williams (1993) and Bravoco and Yadav (1985) have reported on its use in developing manufacturing models applications.

\section{The distribution centre}

The operational processes of a generic supplier distribution centre were studied and modelled using IDEFO (Figure 7). The model has two main streams: the order process (demand management) and the warehouse operations process. Based on data collected from managers at the data collection stage, the main warehouse functions were categorised as inbound planning, tipping, storing, order picking, dispatch planning and dispatch.

Figure 7. IDEFO Model of Distribution Centre Operations

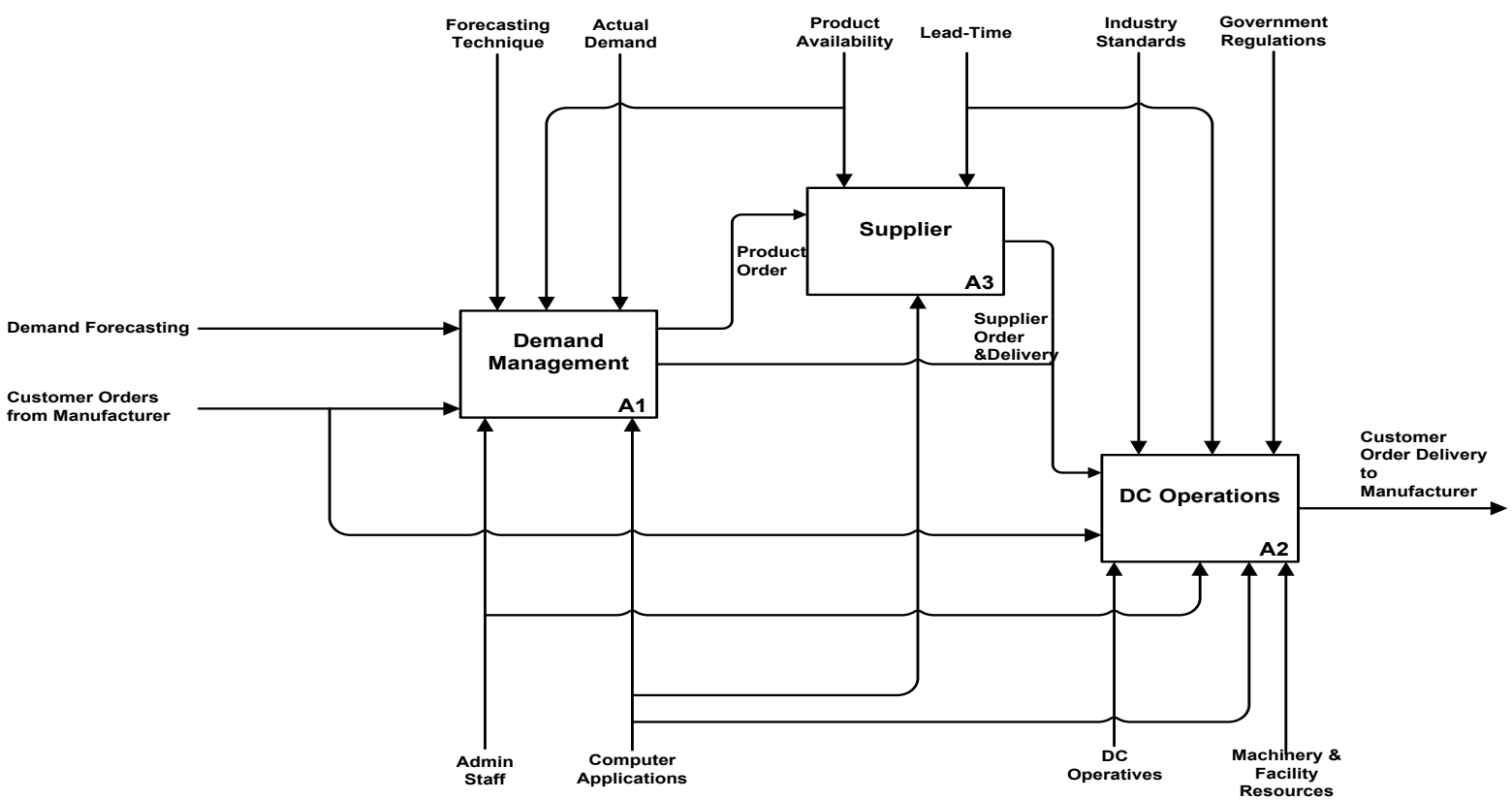

\section{The manufacturing plant}

Studying a plastic packaging manufacturer that uses the distribution centre, IDEF0 was also used to create a detailed conceptual model of the manufacturing process (see Figure 8 ). The production system begins with the arrival of orders from the customer (here, the distribution centre), initiating a 'pull demand' through the production plant. Product items can take a number of possible routes, each having their own individual characteristics, made up of attributes such as height, thickness, quantity needed and extrusion weights, which need to be specifically tailored to meet the individual items' requirements. 
Figure 8. IDEFO Model of Manufacturing Centre Operations

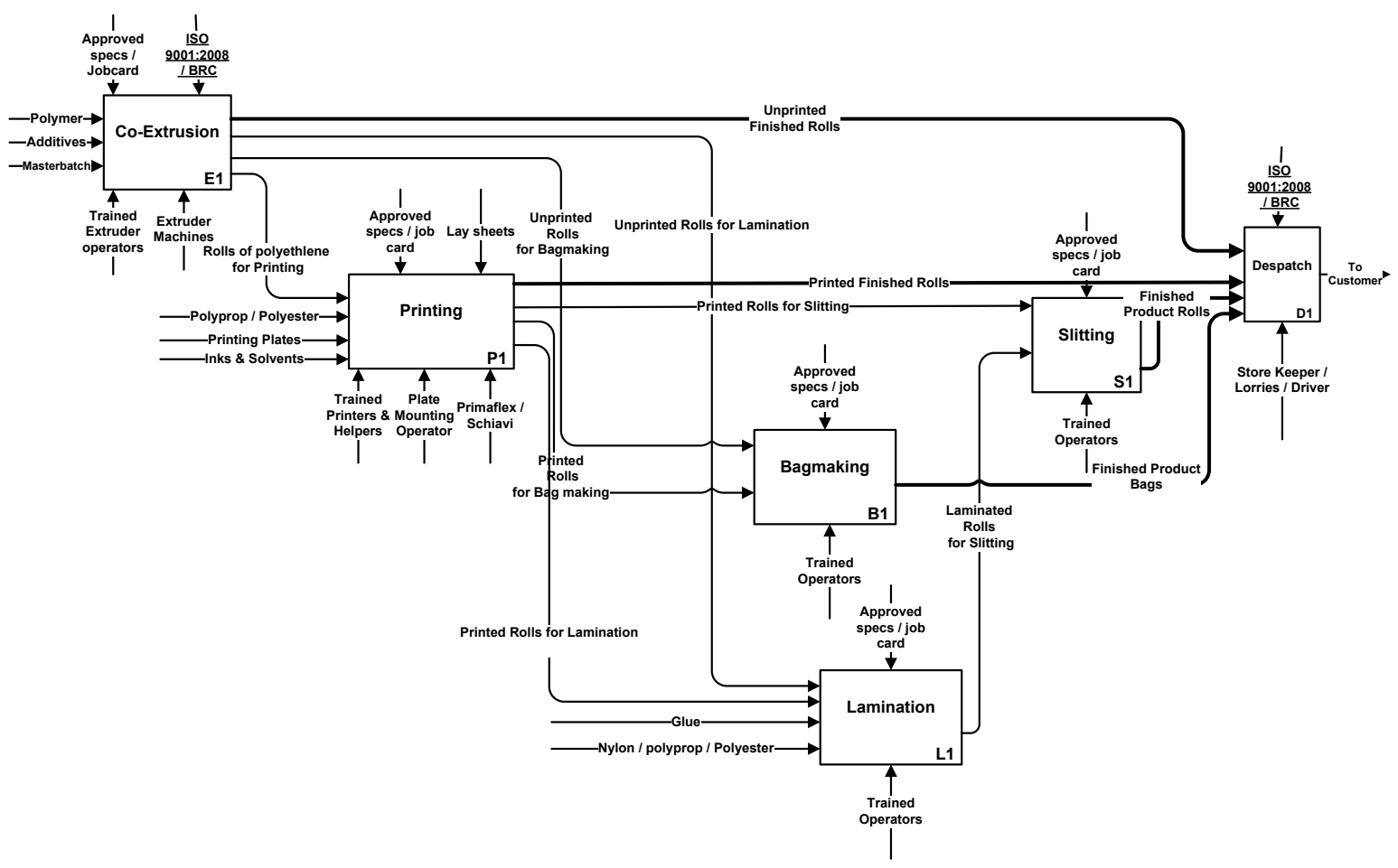

\section{Optimisation agent}

The web-based application of the framework increases the accessibility of the simulation model for students, resulting in increased user playing time. Using the simulation requires that the user makes appropriate decisions in relation to the next actions, but the uncertainty and variability of the supply chain field, which are reflected in the simulation model's tools, makes it very hard for them to make the right decisions. The optimisation agent is integrated in the engine part of the simulation tool to help users make the optimum decisions, or choose from a set of optimum solutions for the current situation. To allow users to benefit from their prior decisions, the agent has a built-in memory that records each case and the solution chosen to assist the user in solving similar cases in the future.

Cases such as warehouse replenishment, seller selection and other decisions that need to be made in the supply chain simulation system, are defined in a formal format that is sent to the optimisation agent, which then uses a genetic algorithm to search for the optimum solution according to a certain set of constraints represented in the rule-base. The case definition and the solution produced are compared with the previous cases stored in the case-base to confirm or modify the solution. After confirming the solution the case-base is updated with the new case-solution pair, and the solution produced is sent to the client through the relevant services and protocols.

\section{Web-enabling application}

The proposed web-based simulation portal structure consists of a client site and a server site connected over the web by TCP/IP protocol.

\section{Server site}

The server site (Figure 9) houses the main structure of the system, which consists of the web server, simulation tool, controller, content management system and database, and listener each of which is described briefly below. 


\section{Web server}

The web server's main purpose is to host the main web sites responsible for the portal, for managing client requests and for data storage with communications managed via the hypertext transfer protocol (HTTP).

\section{Simulation tool}

A professional simulation toolkit is employed to serve the framework's considerable simulation requirements: its range of simulation capabilities is considered as one of this framework's most important advantages over the other web-based simulation tools, which run their simulation tools on clients' machines thereby limiting their simulation capabilities. The tool's main purpose is to run the simulation model according to the client's requirements and decisions (Figures 7 and 8 ) and save the results to be transferred later back to the client.

\section{Controller}

This module is responsible for translating client's requests as commands to the simulation tool. The inputs from the client side are applied through the controller to the simulation tool, and the outputs are then transferred back to the client via the web server using the TCP/IP protocol. The controller uses a shared communication space via which data and commands are exchanged with the simulation tool, and two types of commands: to exchange data by applying inputs and receiving outputs to/from the simulation tool and to execute the simulation tool's options and services.

\section{Content management system and database}

The content management system, built using the four-layer hierarchy method (as illustrated in Figure 9) is used to control accessibility to the system and simulation model. This system differs between the two main user types; TLE instructors and students, giving the former controlling options such as assigning models to users and modifying models, and allowing the latter to run their assigned models and check their results. Log-in to this system is via a username and password interface (as shown in Figure 10). The content management system is supported by a database management system that stores registered users' details, assigned simulation models and the privileges assigned to different types of users.

\section{Listener}

The listener module is the interface between the client and the web server: it 'listens' for client requests and passes them to the web server to be managed by the controller. The client applies requests via a graphical user interface, which are then encoded and sent to the server over the web via the TCP/IP protocol.

Figure 9. Server Site System Structure

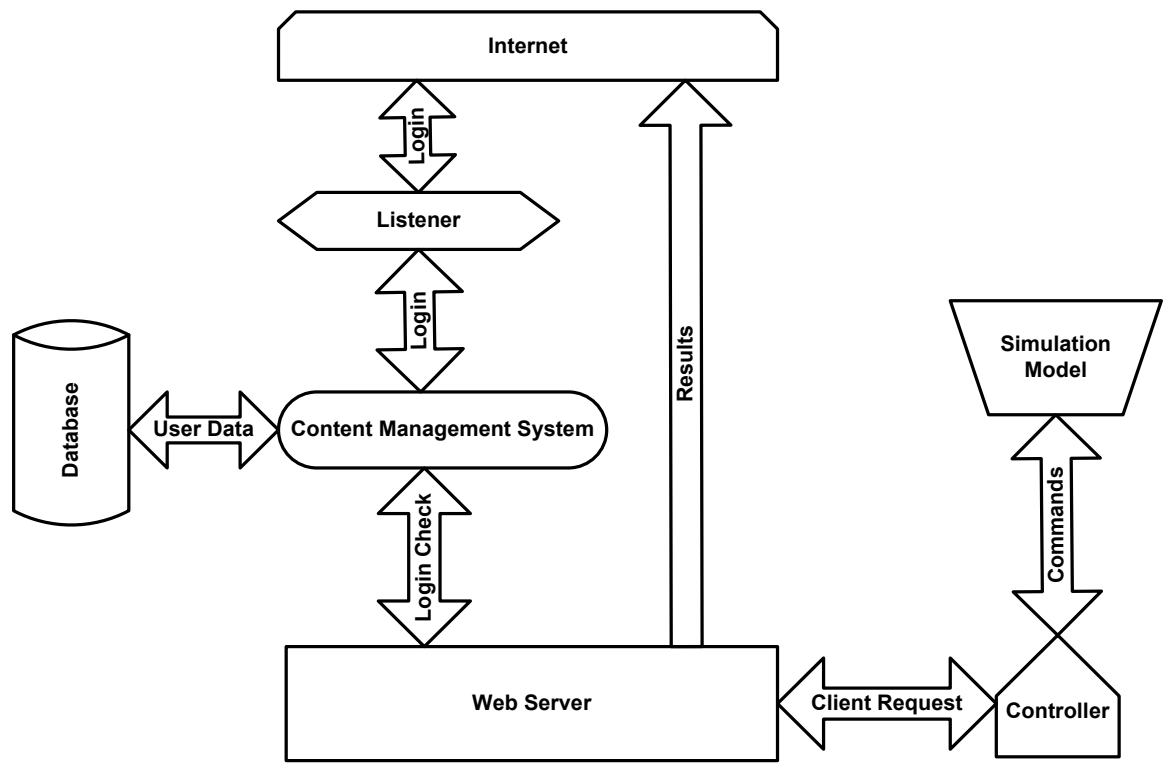


Figure 10. Client Graphical User Interface

\section{Web-Based Simulation Portal}
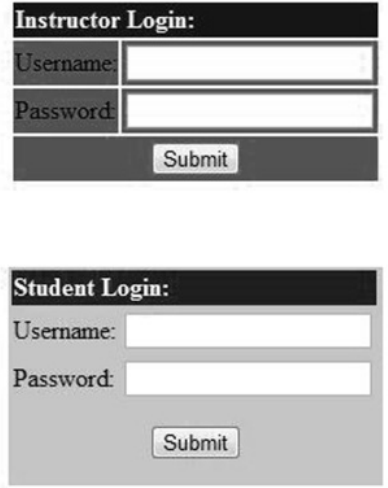

Using this portal the Instructors can manage the accessibility for the students over the simulation models and students can run, control, analyze and compare different simulation models remotely.

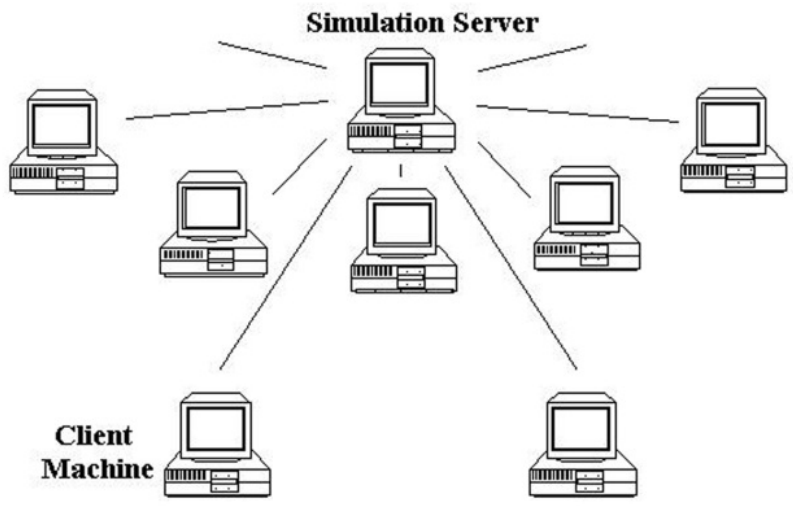

\section{Client site}

The client site represents the part of the system that runs on the client's machine, and consists of two main parts - a GUI and a translator module (which are illustrated in Figure 11) - described below.

Figure 11. Client Site System Structure

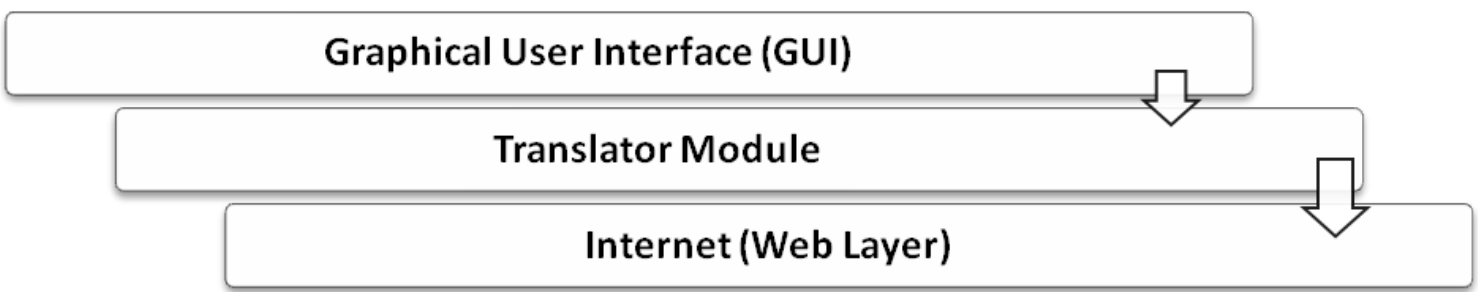

\section{Client graphical user interface (GUI)}

This interface receives client's commands and transfers them to the server, and displays the results received from the server back to the client. The main software engineering capabilities taken into account in building this interface are usability, accessibility and reliability. Clients can process many operations using this interface, including login to the system, controlling the simulation model, applying inputs and checking the results, comparing two simulation models and passing results from one model to other (Figure 12). One of the main benefits of this interface is to facilitate clients' access to the distributed simulation systems.

\section{Translator}

The translator is a module that runs in the background and encodes all actions and commands from all types of users - students and instructors - so they can be understood by the controller on the server site. When the client/ student chooses to run a simulation model, the translator encodes these actions as a running command then sends them over the web. 
Figure 12. User's Operations Scheme

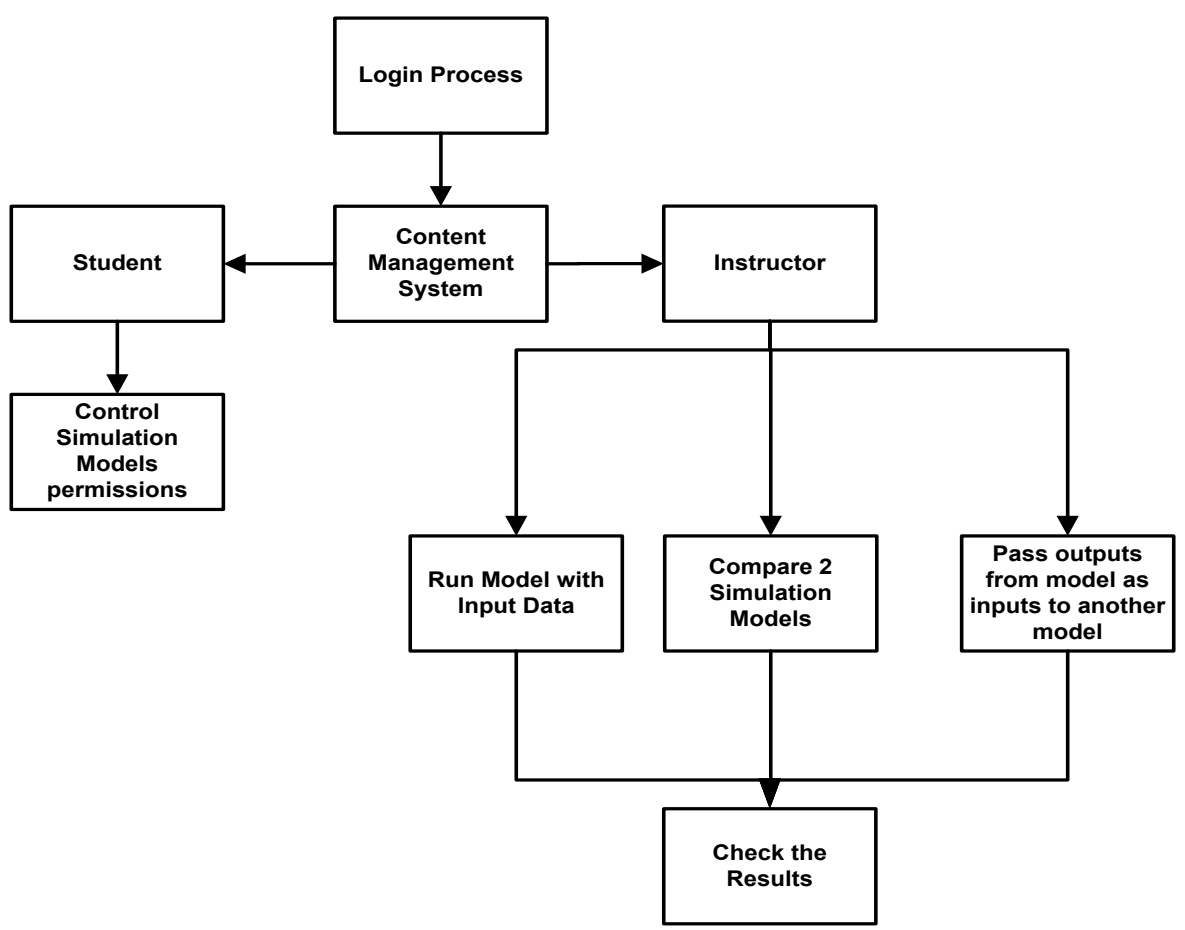

\section{Conclusions}

Ireland's knowledge-driven economy imposes pressures on universities and teachers to adopt novel and effective learning techniques. Educational institutions can no longer ignore the close relationship that today's third level education students have with the technological world.

This paper presents the development of a conceptual framework for creating an interactive eSimulation web portal that enables students to get immediate feedback on their decisions as generated by a simulation model equipped with an integrated solution optimiser. The main focus of the study was to build a user-friendly environment that develops a comprehensive supply chain simulation model as a base engine on a server that is linked to a client agent (an application to be used by the user/student on a web-browser) through the TCP/IP protocol. Information and contents are handled using a relational database system and content management system, allowing students to access their work via portal both in class and also remotely from home.

While developing the portal framework, it was found that using a web-based platform offers several advantages: (1) portals are less expensive than simulation software packages, (2) they are easy to access from anywhere (i.e. college or home), (3) teachers can be given higher authorisations (levels of access) to manage their students' work, and (4) instructors can assign different levels of tasks and assignments to various groups of students, and customise system input and outputs accordingly.

Using the portal, students can learn by experimenting with the changing parameters and examining the outcomes of different scenarios, and can also share the output of their models or inter-connect their results. In the knowledge base considered in this paper, supply chain simulation creates an animated experience for better understanding the impacts of uncertainty, complexity and risks within supply chain networks.

\section{Research limitations}

This eSimulation framework is at a conceptual phase, with a pilot portal in development. To validate its applicability and contribution to the TLE learning process, the portal needs to be run in a classroom environment, so that primary data can be collected and analysed. 


\section{Future research}

The portal interface has the potential to be used within other modules that have similar multiple criteria decisions. A supply chain management game (called AUSUM, for Automobile Supply Chain Management Game) based on the proposed framework is currently being developed.

\section{Acknowledgements}

The authors would like to thank the Irish Research Council (IRC) for their contribution to funding this research.

\section{References}

Aguilar-Savén, R.S. (2004). 'Business process modelling: Review and framework'. International Journal of Production Economics, 90(2), 129-149.

Bicen, H., Ozdamli, F., Ertac, D., Tavukcu, T., Arap, I. and Terali, M. (2010). 'Education needs of teacher candidates towards web based collaborative learning studies'. Procedia-Social and Behavioral Sciences, 2, 5876-5880.

Bond, A. (2002). 'Learning music online: An accessible learning program for isolated students' in: Authority, A.N.T. (ed) Leabrook: National Centre for Vocational Education Research (NCVER).

Bravoco, R.R. and Yadav, S.B. (1985). 'A methodology to model he functional structure of an organization'. Computers in Industry, 6, 345-361

Breena, S., Cleary, J. and O'Shea, A. (2009). 'An investigation of the mathematical literacy of first year third-level students in the Republic of Ireland'. International Journal of Mathematical Education in Science and Technology, 40, 229-246.

Burstein, R.A. and Lederman, L.M. (2001). 'Using wireless keypads in lecture classes'. The Physics Teacher, 39, 8-11.

Busby, J. and Williams, G. (1993). 'The value and limitations of using process models to describe the manufacturing organization'. The International Journal of Production Research, 31, 2179-2194.

Byrne, J., Heavey, C. and Byrbe, P.J. (2010). 'A review of Web-based simulation and supporting tools'. Simulation Modelling Practice and Theory, 18, 253276.

Caldwell, J.E. (2007). 'Clickers in the large classroom: Current research and best-practice tips'. Life Sciences Education, 6(1), 9-20.

Caridi, M., Crippa, L., Perego, A., Sianesi, A. and Tumino, A. (2010). 'Do virtuality and complexity affect supply chain visibility?'. International Journal of Production Economics, 127, 372-383.

Chan, E.A. (2009). 'Reflecting on the essence of our problem-based learning discussions: The importance of faculty development and our continuous quest for applications of problem based learning'. Kaohsiung Journal of Medical Science, 25, 276-281.

Christopher, M. (2005). Logistics and supply Chain Management: Creating Value-added Networks, Pearson education.

Christopher, M. (2010). Logistics and Supply Chain Management, Financial Times/Prentice Hall.

Christopher, M. and Lee, H. (2004). 'Mitigating supply chain risk through improved confidence'. International Journal of Physical Distribution \& Logistics Management, 34, 388-396.

Clancy, P. and Kehoe, D. (1999). 'Financing thirdlevel students in Ireland'. European Journal of Education, 34, 43-57.

Colquhoun, G.J., Baines, R.W. and Crossley, R. (1993). 'A state of the art review of IDEFO'. International Journal of Computer Integrated Manufacturing, 6 , 252-264.

Cousins, P. D. (2002). 'A conceptual model for managing long-term inter-organisational relationships'. European Journal of Purchasing \& Supply Management, 8, 71-82.

Crowe, J., Mahfouz, A., Arisha, A. and Barrett, F. (2010). 'Customer Management Analysis of Irish Plumbing \& Heating Distribution System: A Simulation Study'. 2nd International SIMUL Conference, Nice.

Davis, D.M., Gottschalk, T.D. and Davis, L.K. 'Highperformance computing enables simulations to transform education'. Simulation Conference, 2007 Winter, 9-12 Dec. 2007, 2336-2343.

Davis, T. (1993). 'Effective supply chain management'. Sloan Management Review, 34(4), 35-35.

Dekanter, N. (2004). 'Gaming redefines interactivity for learning'. TechTrends, 49(3), 26-31.

Dewey, J. (1938). Experience and Education, New York, Collier Macmillan Publishers.

Education, D.O. (1995). 'Charting our education future white paper on education' in: Department Of 
Education And Science (ed), Dublin: Stationery Office.

Ferdig, R.E., Coutts, J., Dipietro, J. and Lok, B. (2007). 'Innovative technologies for multicultural education needs'. Multicultural Education \& Technology Journal, 1, 47-63.

Fishwick, P.A. 'Web-based simulation: Some personal observations'. Simulation Conference, 1996. Proceedings. Winter, 1996, 772-779.

Fowler, C.H. and Mayes, J.T. (2000). 'Learning relationships from theory to design'. Association of Learning Technology Journal, 7(3), 6-16.

Gaba, D.M. (2007). 'The future vision of simulation in healthcare'. Simulation in Healthcare, 2, 126-135.

Garrison, R.D. and Kanuka, H. (2004). 'Blended learning: Uncovering its transformative potential in higher education'. Internet and Higher Education, 7, 95-105.

Goffin, K., Lemke, F. and Szwejczewski, M. (2006). 'An exploratory study of 'close' supplier-manufacturer relationships'. Journal of Operations Management, 24, 189-209.

Holweg, M., Disney, S., Holmström, J. and Småros, J. (2005). 'Supply chain collaboration: Making sense of the strategy sontinuum'. European Management Journal, 23, 170-181.

Huin, S., Luong, L. and Abhary, K. (2002) 'Internal supply chain planning determinants in small and mediumsized manufacturers'. Management, 32, 771-782.

Hung, W., Kucherenko, S., Samsati, N. and Shah, N. (2004) 'A flexible and generic appraoch to dynamic modelling of supply chains'. Journal of Operational Reesearch Society, 55, 801-813.

Ismail, K., Abo-Hamad, W. and Arisha, A. 'Integrating balanced scorecard and simulation modeling to improve emergency department performance in Irish hospitals'. Winter Simulation Conference (WSC), Proceedings of the 2010, 5-8 Dec. 2010, 2340-2351.

lyer, A.V., Seshadri, S. and Vasher, R. (2009). Toyota Supply Chain Management, New York, McGraw-Hill.

Jüttner, U., Christopher, M. and Baker, S. (2007). 'Demand chain management-integrating marketing and supply chain management'. Industrial Marketing Management, 36, 377-392.

Katsaliaki, K., Mustafee, N. and Kumar, S. (2014). 'A game-based approach towards facilitating decision making for perishable products: An example of blood supply chain'. Expert Systems with Applications, 41, 4043-4059.

Khalifa, M. and Lam, R. (2002). 'Web-based learning: Effects on learning process and outcome'. Education, IEEE Transactions on, 45, 350-356.
Knight, J.K. and Wood, W.B. (2005). 'Teaching more by lecturing less'. Cell Biology Education, 4, 298-310.

Lambert, D., Cooper, M. and Pagh, J. (1998). 'Supply chain management: implementation issues and research opportunities'. International Journal of Logistics Management, 9, 1-20.

Lambert, D. and Pohlen, T. (2001). 'Supply chain metrics'. International Journal of Logistics Management, 12, 1-20.

Laurillard, D. (1998). 'Multimedia and the learner's experience of narrative'. Computers \& Education, 31, 229-242.

Longo, F. and Mirabelli, G. (2008). 'An advanced supply chain management tool based on modeling and simulation'. Computers \& Industrial Engineering, 54, 570-588.

Mahfouz, A., Ali Hassan, S. and Arisha, A. (2010). 'Practical simulation application: Evaluation of process control parameters in twisted-pair cables manufacturing system'. Simulation Modelling Practice and Theory, 18, 471-482.

McKee, J.A. and Lantz, K.A. (2009). 'The ABC simulator: A new approach to teaching traditional topics'. Management Accounting Quarterly, 10(4), 28-33.

McKeever, S. (2003). 'Understanding Web content management systems: Evolution, lifecycle and market'. Industrial Management \& Data Systems, 103, 686-692.

Moore, D.E., Green, J.S. and Gallis, H.A. (2009). 'Achieving desired results and improved outcomes: Integrating planning and assessment throughout learning activities'. Journal of Continuing Education in the Health Professions, 29, 1-15.

Murphy, S., Hartigan, I., Walshe, N., Flynn, A.V. and O'Brien, S. (2011). 'Merging problem-based learning and simulation as an innovative pedagogy in nurse education'. Clinical Simulation in Nursing, 7, e141-e148.

O'Neill, G., Moore, S. and McMullin, B., (eds) (2005). 'Teaching and learning activities: Expanding the repertoire to support student learning' in: (Hea), H.E.A.I. (ed) Dublin: All Ireland Society for Higher Education (AISHE).

Page, E.H., Griffin, S.P. and Rother, L. 'Providing conceptual framework support for distributed Webbased simulation within the high level architecture'. Proceedings of SPIE: Enabling Technologies for Simulation Science II, 1998 Orlando. Citeseer.

Page, E.H. and Opper, J.M. Observations on the complexity of composable simulation. 1999, IEEE, 1, 553-560.

Palmer, P.J. (2010). The courage to teach: Exploring the inner landscape of a teacher's life. Wiley.com. 
Prince, M. (2004). 'Does active learning work? A review of the research'. Journal of Engineering Education, 93, 223-231.

Proserpio, L. and Gioia, D.A. (2007). 'Teaching the virtual generation'. Academy of Management Learning \& Education, 6, 69-80.

Rainee, J.D. and Guyton, E. (1994). 'Developing a constructivist teacher education program: The policy-making stage'. Journal of Teacher Education, 45, 140-151.

Reichenthal, S.W. 'Re-introducing Web-based simulation'. Simulation Conference, 2002. Proceedings of the Winter, 8-11 Dec. 2002, 1, 847-852.

Rein, G.L., McCue, D.L. and Slein, J.A. (1997). 'A case for document management functions on the Web'. Communications of the ACM, 40(9), 81-89.

Ross, D.T. (1977). 'Structured analysis (SA): A language for communicating ideas'. Software Engineering, IEEE Transactions on, 16-34.

Ross, D.T. and Schoman JR, K.E. (1977). 'Structured analysis for requirements definition'. Software Engineering, IEEE Transactions on, 6-15.

Rossetti, M.D., Miman, M. and Varghese, V. (2008). 'An object-oriented framework for simulating supply systems'. Journal of Simulation, 2, 103-116.

Ruben, B. (1999). 'Simulations, games, and experiencebased learning: The quest for a new paradigm for teaching and learning'. Simulation \& Gaming, 30, 498-505.

Savery, J. and Duffy, T. (1996). 'Problem based learning: An instructional model and its constructivist framework'. Constructivist learning environments: Case studies in instructional design, 135-148.

Schleicher, A. (2003). 'Progress in education: Studying the signs'. Organisation for Economic Cooperation \& Development. The OECD Observer, 239: Sep, 33.

Shen, H., Wall, B., Zaremba, M., Chen, Y. and Browne, J. (2004). 'Integration of business modelling methods for enterprise information system analysis and user requirements gathering'. Computers in Industry, 54, 307-323.

Skills, D.O.E.A. (2012). Key Statistics 2011/2012, available from: <http://www.education.ie/en/ Publications/Statistics/Key-Statistics-2012.pdf>
[Accessed on 12th June 2013].

Skinner, B.F. (1954). 'The science of learning the art of science'. Harvard Educational Review, 24, 86-97.

Spekman, R., Kamauff J.R. and Myhr, N. (1998). 'An empirical investigation into supply chain management'. Management, 28, 630-650.

Squire, K. (2003). 'Video games in education'. International Journal of Intelligent Simulations and Games, 2(1), 49-62.

Stefanou, C., Stolk, J., Prince, M., Chen, J. and Lord, S. (2013). 'Self-regulation and autonomy in problemand project-based learning environments'. Active Learning in Higher Education, 14, 109-122.

Syrjakow, M., Berdux, J. and Szczerbicka, H. 'Interactive Web-based animations for teaching and learning'. Simulation Conference Proceedings, 2000. Winter, 2000, 2, 1651-1659.

Tobail, A., Crowe, J. and Arisha, A. (2010). 'Web-based supply chain simulation: An integrated approach'. ICERI 2010, Madrid.

Towill, D. (1991). 'Supply chain dynamics'. International Journal of Computer Integrated Manufacturing, 4, 197-208.

Van der Vorst, J. and Beulens, A. (2002). 'Identifying sources of uncertainty to generate supply chain redesign strategies'. Management, 32, 409-430.

Van der Zee, D.J. and Slomp, J. (2009). 'Simulation as a tool for gaming and training in operations management - A case study'. Journal of Simulation, 3, 17-28.

Vidgen, R., Goodwin, S. and Barnes, S. Web Content Management, 2001, 465-480.

Von Glasersfeld, E. (1989). 'Cognition, construction of knowledge, and teaching'. Synthese, 80, 121-140.

Wiedemann, T. (2001). 'Simulation application service providing (SIM-ASP)'. Simulation Conference, 2001. Proceedings of the Winter, 2001, 1, 623-628.

Wilding, R. (1998) 'The supply chain complexity triangle'. Management, 28, 599-616.

Yingping, H. and Madey, G. (2005) 'Autonomic Webbased simulation'. Simulation Symposium, 2005. Proceedings. 38th Annual, 4-6 April 2005, 160-167.

Zhang, C., Thompson, S. and Miller, C. (2011). 'A review of simulation-based interprofessional education'. Clinical Simulation in Nursing, 7, e117-e126. 